\title{
Report of the ASFA Apheresis Registry on Muscle Specific Kinase Antibody Positive Myasthenia Gravis
}

Chisa Yamada, M.D. ${ }^{1}$, Huy P. Pham, M.D. ${ }^{2}$, Yanyun Wu, M.D. ${ }^{3,4}$, Laura Cooling, M.D. ${ }^{1}$, Haewon C. Kim, M.D. ${ }^{5}$, Shanna Morgan, M.D. ${ }^{6}$, Joseph Schwartz, M.D. ${ }^{7}$, Jeffrey L. Winters, M.D. ${ }^{8}$, Edward C.C. Wong, M.D. ${ }^{9}$

${ }^{1}$ Division of Transfusion Medicine

Department of Pathology

University of Michigan

Ann Arbor, MI

${ }^{2}$ Division of Laboratory Medicine

Department of Pathology

University of Alabama at Birmingham

Birmingham, AL

${ }^{3}$ Bloodworks Northwest

Seattle, WA

${ }^{4}$ Division of Laboratory Medicine

Yale Medical School

New Haven, CT

${ }^{5}$ Division of Transfusion Medicine

Department of Pathology

Children's Hospital of Philadelphia

Philadelphia, PA

${ }^{6}$ American Red Cross

St. Paul, MN

${ }^{7}$ Division of Transfusion Medicine

Department of Pathology \& Cell Biology

Columbia University

Ney York, NY

${ }^{8}$ Division of Transfusion Medicine

Department of Laboratory Medicine and Pathology

Mayo Clinic

Rochester, MN

${ }^{9}$ Division of Laboratory Medicine

Center for Cancer and Blood Disorders

Children's National Medical Center

Departments of Pediatrics and Pathology

This is the author manuscript accepted for publication and has undergone full peer review but has not been through the copyediting, typesetting, pagination and proofreading process, which may lead to differences between this version and the Version record. Please cite this article as doi:10.1002/ jca.21454. 
George Washington School of Medicine and Health Science Washington DC

Address for correspondence:

Chisa Yamada, M.D.

University of Michigan

Blood Bank / Transfusion Medicine

Department of Pathology

1500 E. Medical Center Dr.

SPC 5054, 2F225 UH

Ann Arbor, MI 48109-5054

Tel: (734) 936-6776

Fax: (734) 936-6888

E-mail: yamadac@med.umich.edu
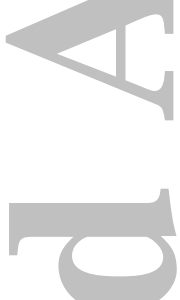

Running head: ASFA apheresis registry for MuSK MG 


\begin{abstract}
Background: Anti- muscle specific kinase antibody positive (MuSK Ab) myasthenia gravis (MG) patients are known to have different clinical course compared to acetylcholine receptor $\mathrm{Ab}$ positive MG patients. Therapeutic plasma exchange (TPE) has been reported to be effective; however, little is known of the response and of TPE procedural information. An ASFA Apheresis Registry was developed to analyze those data. Methods: The study collected detailed de-identified patient data, TPE procedures, and treatment outcome/complications. Collected data was described in aggregate. Results: A total of $15 \mathrm{MuSK}$ Ab MG patients with exacerbation of MG symptoms, 13 females/2 males, median age 44, were investigated. Thirty TPE courses (median 5 procedures/course, total 145 procedures) were evaluated. All TPE procedures were performed with citrate anticoagulation, 1-1.25 plasma volume exchange in 100\% fluid balance, and $90 \%$ of courses used only albumin as replacement. Calcium was added to albumin or given orally as needed. TPE was performed every other day in 55\% of courses. Adverse events occurred in $3.4 \%$ of procedures. Ten patients (67\%) experienced relapses within a median of 7 weeks. Objective symptoms were resolved in more than $75 \%$ of courses. Overall subjective improvement rates were 94.1\%/93.3\% after 3/4 TPE procedures, respectively. Thirty-one percent of patients responded poorly with minimal recovery. Conclusion: Overall subjective improvement was seen up to $94 \%$ of patients after one course of TPE. Some patients were poorresponders. Five TPE may be adequate for initial course with additional TPE as needed. Based upon this preliminary data, we will modify our future data collection.
\end{abstract}

Key Words: ASFA apheresis registry, Therapeutic plasma exchange, MuSK MG 


\section{Introduction}

Approximately $20 \%$ of Myasthenia gravis (MG) patients do not have acetylcholine receptor (AchR) antibodies (Abs) defining a conditions known as "sero-negative" MG. [1] However, additional auto-antibodies to other neuromuscular junction proteins have been recently discovered in these patients such as antibodies directed against muscle specific kinase (MuSK) [1], the muscle proteins titin or ryanodine [2], and lipoprotein receptor-related protein 4 (Lrp4) [3]. Other potential antigenic targets include neural agrin, which binds to Lrp4, and CoIQ which is a part of acetylcholinesterase complex [4].

Anti-MuSK Ab positive MG (MuSK MG) patients are often reported to have a different clinical course compared to anti-AchR Ab positive MG (AchR MG) patients. The repeatedly reported features of MuSK MG patients compared to AchR MG patients are listed in Table 1. Therapeutic plasma exchange (TPE) is reported to be one of the effective treatments for MuSK MG patients. Maintenance TPE has also been reported to be beneficial, resulting in patients having little or no symptoms for protracted periods of time. [5] However, little is known how the clinical response relates to the performance of TPE, including total number of procedures, frequency of the procedure, or replacement fluid during a course of treatment.

The multi-institutional apheresis registry was developed by the American Society for Apheresis (ASFA) Research subcommittee. The aim is to collect and analyze outcome, safety, and practice information related to apheresis. We collected data from the patients with MuSK MG who received TPE treatment as a part of the apheresis registry and report the first data summary. 


\section{Materials and Methods}

The ASFA Apheresis Registry is a multi-institutional database which currently has data from 17 medical centers / facilities from across the United States. For this first report for MuSK MG registry, New York-Presbyterian Hospital-Columbia University Medical Center (New York, NY), University of Michigan (Ann Arbor, MI), and Yale New Haven Hospital (New Haven, CT) identified MuSK MG patients and participated in data entry. These facilities had obtained their Institutional Review Board (IRB) approval and a Data Usage Agreement to enter the detailed deidentified patient demographic and clinical information, apheresis procedural information, treatment schedules, treatment outcome and procedural-complications into a Research Electronic Data Capture (REDCap) database, both retrospectively and prospectively. The REDCap database is an electronic data capture tools hosted at Children's National Medical Center and is a secure, web-based application designed to support data capture for research studies, providing 1) an intuitive interface for validated data entry; 2) audit trails for tracking data manipulation and export procedures; 3) automated export procedures for seamless data downloads to common statistical packages; and 4) procedures for importing data from external sources. [6]

Included in the database were MG patients with anti-MuSK Ab who received a course of TPE due to symptomatic exacerbation. Patients receiving TPE treatment at a frequency of less than one month were considered to be in a course of treatment. If patients experienced a relapse and received another course of TPE treatment, the data for that course was entered separately. Although some patients received maintenance TPE between each course with more frequent TPE for symptomatic exacerbation, the data during the maintenance TPE was not collected. 
The demographic data collected includes gender, race, age at diagnosis, and age at first TPE. The TPE procedural data collected includes total number and frequency of procedures in one TPE course, plasma volume exchanged, type of replacement fluid, type of anticoagulant, type of access, use of RBC prime, the type of electrolyte (calcium, magnesium, and potassium) supplementation and adverse events.

The clinical data collected includes symptoms, medications used, thymectomy, antibody specificity (AchR $\mathrm{Ab}$ and MuSK Ab), Edrophonium test results, electromyography (EMG) abnormalities, single fiber electromyography (SFEMG) abnormalities, and relapse. Seven objective symptoms (diplopia, ptosis, dysarthria, dysphagia, limb weakness, shortness of breath [SOB], and intubation) were reported at onset of the disease, before and after each TPE, within one month, and 1-3 and 3-6 months after the last TPE when the data was available in the clinical record. Since improvement was evaluated on subsequent patient encounter, the evaluation of the last TPE procedure in a course was not available. The objective symptoms found in each patient's clinical records at each time point retrieved by site study team member(s) at each facility were entered to the data base. The subjective symptomatic improvement found in clinical records written by apheresis physicians or neurologists at each time point were retrieved and evaluated by site study team member(s) at each facility and categorized into no, mild, moderate, marked, and full improvement. Blood pressure (BP) before and after each TPE procedure was also recorded when available. The medications (prednisone, hydrocortisone, methylprednisone, pyridostigmine, IVIG, rituximab, azathioprine, mycophenolate mofetil, and/or cyclosporine) before and after a course of TPE were also recorded when available. 
Analysis for all collected data was performed in aggregate. The descriptive analysis was mainly used with mean, median ratio and t-test when applicable. For the t-test, a p-value of less than or equal to 0.05 was considered significant.

\section{Results}

\section{Demographic data}

As of 9/1/15, a total of $15 \mathrm{MuSK}$ Ab positive MG patients from 3 centers treated for exacerbation of MG symptoms between 2006 and 2013 had been entered into the registry. Thirteen patients were female $(86.7 \%)$ and 2 patients were male $(13.3 \%)$. Seven patients were Caucasian, 2 patients were African American, 1 patient was Asian, and 5 patients were of unknown ethnicity. The age at diagnosis was stratified by decade in life with 1, 3, 2, 4, 2 and 3 patients in the $2^{\text {nd }}, 3^{\text {rd }}, 4^{\text {th }}, 5^{\text {th }}, 6^{\text {th }}$ and $7^{\text {th }}$ decade of life respectively. Median age at diagnosis was 43 (14-66) years old, and median age at first TPE was 43 (16-67) years old.

\section{TPE procedures}

Summary of TPE procedural data is shown in Table 2 (occurrence rates are based on number of courses in Table 2A, based on number of procedures in Table 2B). Thirty TPE courses (total of 145 procedures) were evaluated. All TPE procedures/courses used a 1-1.25 plasma volume exchange with $100 \%$ fluid balance and full rinse back. Anticoagulant citrate dextrose solution A (ACD-A) was used as the procedural anticoagulant. The number of TPE procedures per course ranged from 2 to 10 procedures, with a median of 5 procedures overall. TPE frequency was reported in 29 courses; with TPE being performed most frequently every 
other day in 16 of $29(55.2 \%)$ courses. The data for type of access was entered in 28 courses; peripheral veins were used in 14 of $28(50.0 \%)$ courses and a central venous catheter was placed in 13 of $28(43.3 \%)$ courses, primarily in the internal jugular vein. Switching access from one type to another in one course was not reported. Replacement fluid was 5\% albumin only in 133 of $145(91.7 \%)$ procedures in 27 courses at all facilities and 5\% albumin and normal saline in 12 of $145(8.3 \%)$ procedures in 3 courses with albumin: normal saline ratio of 5:1, 4:1, 5:2 and 1:1 at one facility. Calcium supplementation was reported in 136 procedures; calcium was added into albumin in 114 of $136(83.8 \%)$ procedures in 25 courses at two facilities per protocol; and given orally in one of $136(0.7 \%)$ procedures for citrate toxicity in one course. None of the patients received potassium or magnesium supplementation and none required RBC priming. Adverse events were reported in 5 of $145(3.4 \%)$ procedures.

\section{Clinical course}

One of the 15 patients also had anti-AchR $\mathrm{Ab}$ in addition to anti-MuSK $\mathrm{Ab}$ and underwent thymectomy. A total of 3 patients (20\%) underwent thymectomy. Positive Edrophonium test was reported in 2 patients (13.3\%), abnormal EMG and SFEMG were reported in $2(13.3 \%)$ and 5 patients (33.3\%) respectively. Pre-procedure BP was reported in 127 procedures, and post-procedure BP was reported in 23 procedures. Pre-procedure systolic/diastolic pressure ranged $85-183 / 45-107 \mathrm{mmHg}$ and $92-148 / 62-90 \mathrm{mmHg}$ postprocedure respectively.

Table 3 shows percentage of objective symptoms at onset and immediately prior to each TPE course, and the full recovery percentage of each symptom after a course of TPE. Immediately prior to each TPE course $(n=30)$, each symptom was reported to be present in more 
than $40 \%$ of courses except intubation. Within the patients who had each symptom before TPE treatment, the full recovery percentage of symptom was more than $75 \%$.

Subjective improvement was evaluated before each subsequent TPE; therefore, subjective findings were typically not available after the last TPE in one treatment course. Because most patients received 5 TPE per course, subjective improvement by procedure is primarily limited to the first 4 procedures: two patients received more than 5 TPE. Table 4 shows total number of TPE procedures in one course and final subjective improvement level in reported 25 TPE courses. None, mild, moderate, marked and full improvement were found in 3, 6, 5, 7, 4 courses respectively. Table 5 shows the degree and percentage of improvement after each TPE procedure in up to 25 reported treatment courses. Any degree (mild to full) of improvement was achieved in $94.1 \%$ and $93.3 \%$ with marked improvement of $29.4 \%$ and $40 \%$ after $3^{\text {rd }}$ and $4^{\text {th }} \mathrm{TPE}$ procedure, respectively. Of note, since the numbers of reported courses are different depending on $1^{\text {st }}$ to $>5^{\text {th }}$ TPE, the occurrence percentage of improvement is based on the number of reported courses in each evaluation time.

Pre-procedure medications in 30 courses are listed in Table 6. Before the first TPE, patients were taking prednisone and pyridostigmine in more than $40 \%$ of the courses. None of the patients were receiving methylprednisone and cyclosporine, however, one patient received methylprednisone during the course of TPE most likely on admission to the hospital. The dose of prednisone was reported in 14 courses after a course of TPE and 13 courses within 3 months (Table 7A). The prednisone dose was not changed at either evaluation times in the majority of the treatment courses. The dose of pyridostigmine was reported only in 6 and 5 courses after a course of TPE and within 3 months, respectively (Table 7B). The pyridostigmine dose was decreased at both evaluation times in the majority of the treatment courses. 
Ten patients (67\%) experienced one or multiple relapses within a median of 7 weeks (2 weeks - 18 months) after the last TPE in one course of treatment.

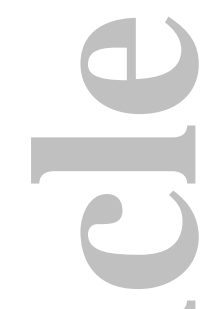

\section{Discussion}

MG is an autoimmune disease and relatively common condition. Treatment regimens such as steroids, immunosuppressives, cholinesterase inhibitors, thymectomy, IVIG and TPE, have decreased mortality rate from MG substantially. However, some patients with "seronegative" MG still experience severe symptoms. Many patients with "sero-negative" MG have anti-MuSK Abs and have been reported to respond well to TPE. [7-11] However; details of TPE procedure and patient clinical responses have not been investigated.

Our demographic data showed female predominance, which is the same as already published reports of MuSK MG patients. However, the highest occurrence age in our study was in the $5^{\text {th }}$ decade of life, which is slightly older than those found in previous reports $\left(3^{\text {rd }}\right.$ and $4^{\text {th }}$ decade of life) [7-11]. This may be due to the limited number of patients in our study. The clinical feature of MuSK MG patients in our study confirmed reported features including low incidence of thymectomy and abnormal EMG, high incidence of more severe symptoms, oculobulbar symptoms, relapse and abnormal SFEMG. [7-11] We included one patient who had both anti-AchR $\mathrm{Ab}$ and anti-MuSK $\mathrm{Ab}$ from our analysis at this time because we could not exclude the symptoms from anti-AchR $\mathrm{Ab}$ and because of limited number of participants. We consider separating those patients in future data analysis.

In our study, we found that five TPE procedures were performed as a course of treatment in 14 of $30(46.7 \%)$ courses. We surmise this is mainly because 5 procedures in one TPE course 
are necessary to assess a patient's initial response to TPE for many neurological diseases in some facilities. This may be based on ASFA's recommendation to typically performed 5-6 procedures for any disease (with few exceptions) and because of institutional protocol. In our study, TPE was performed every other day in $55.2 \%$ of courses; however, we did not stratify TPE frequency based on the severity of symptoms. Further study may be necessary to determine if changes in procedural frequency ameliorate certain symptoms faster than others. Overall, plasma volumes exchanged (1-1.25 of plasma volume), 100\% fluid balance, full rinse back, calcium supplementation and anticoagulation (ACD-A) use were the predominant TPE procedural features in the facilities surveyed. RBC prime for the procedure was not performed in any procedure most likely because all participated patients were adults.

Albumin $(5 \%)$ was used as a replacement fluid in the majority of procedures, with 12 procedures in 3 courses using combination of 5\% albumin and normal saline in variable ratio with $100 \%$ fluid balance. All 3 courses were performed in one facility and the use of normal saline is most likely dependent on that facility's TPE protocol. Since normal saline has only a transient intravascular effect and can cause fluid and protein depletion [12] possibly leading to hypotension, some facilities avoid using normal saline other than priming of the apheresis machine. The post-procedure BP showed variable change compared to pre-procedure BP in those 12 procedures without any clinical symptoms associated with BP; the systolic BP change ranged from -26 to $+20 \mathrm{mmHg}$, the diastolic $\mathrm{BP}$ change ranged from -25 to $+13 \mathrm{mmHg}$. These changes may be significant depending on the patient's condition, therefore, careful observation may be required. However, in 10 procedures in 2 other courses which used only albumin in the same facility, systolic BP change ranged from -9 to $+9 \mathrm{mmHg}$, and diastolic BP change ranged from -10 to $+42 \mathrm{mmHg}$ (from pre-TPE diastolic $\mathrm{BP}$ of $45 \mathrm{mmHg}$ ). The difference of BP change 
between with and without normal saline use is not statistically significant (systolic pressure change $p=0.8364$, diastolic pressure change $p=0.1357$ ).

Incidence of adverse events with TPE varies in the literature between $1.6 \%$ and $25 \%$. [13] Couriel reported overall TPE complication rate of 17\% [14], while Mokrzycki reported a complication rate of 9.7\% [15]. Mokrzycki also reported that hypocalcemia due to citrate was reduced to $1 \%$ with calcium prophylaxis from $9.1 \%$ without prophylaxis. Our study demonstrated an incidence of adverse events of 3.4\%. Two adverse events were citrate toxicity. Calcium supplementation was not given for those two procedures, although it was given orally to treat the symptoms in one procedure. Therefore, citrate toxicity occurred in 2 of 22 procedures (9.1\%) with no calcium supplementation, and $0 \%$ with calcium supplementation, similar to the effect of calcium supplementation on hypocalcemia reported by Mokrzycki. Thus, our study suggests that calcium supplementation may be needed to mitigate citrate toxicity while performing TPE procedures for MuSK Ab positive MG patients.

In regards to response to TPE, more than $75 \%$ of any objective symptoms fully resolved after a course of TPE (Table 3). Overall subjective improvement was also achieved up to 94.1\% of courses (Table 5). The most frequent mild, moderate, marked, and full improvement was found after $2^{\text {nd }}, 3^{\text {rd }}, 4^{\text {th }}$ and $4^{\text {th }}$ TPE procedures, respectively. This observation represents increasing patient symptomatic improvement with increasing number of TPE procedures. Given that patients did not make clinic visits soon after the $5^{\text {th }}$ procedure, we were not able to assess improvement in patient symptomatology after the $5^{\text {th }}$ procedure. However, due to satisfactory improvement rate after the $4^{\text {th }}$ procedure, we surmise that 5 TPE procedures may be an appropriate initial number of TPE procedures in one course and further procedures can be added if the patients' response is poor. 
Four of reported $13(30.8 \%)$ patients had only mild or no subjective improvement after one or two courses of TPE (Patients 1, 3, 14, 15, Table 4). These patients received one or two courses of TPE treatments. These patients may be poor- or non-responders to TPE. Two other patients received more than 5 TPE procedures in 2 of 6 courses (Patient 7) and all 4 courses (Patients 13), probably for poor clinical response. In these 2 patients, the response to TPE progressively diminished over time. These patients initially had marked improvement after their first course but had mild or no improvement after their last treatment course. Therefore, patients may be classified into 3 groups based on their response to TPE; 1. Patients with good sustainable response, 2. Patients with poor response, 3. Patients with diminishing response (initial good improvement, but not sustainable). When the patients are classified in these three groups in our study, the prevalence was $53.8,30.8$, and $15.4 \%$ in group 1,2 , and 3 , respectively. However, additional patients are needed to confirm this observation.

Although the use of maintenance TPE for MuSK MG patients was reported previously [5], the purpose of this study was to evaluate the response to TPE in patients with symptomatic exacerbation; therefore, we did not collect data for maintenance TPE. The patient's symptoms can be stabilized with maintenance TPE; however, mild to moderate relapses are not uncommon even during the maintenance TPE in those patients with anti-MuSK MG.

Only limited number of information for medication dose was entered into REDCap system. However, based on this limited data, TPE may have a potential to decrease prednisone within 3 months and pyridostigmine after a course of TPE. Reducing high dose steroid is important in order to avoid side effects associated with its long-term use. The additional entry of more MuSK MG patients may allow a more detailed analysis of medication reduction after a TPE course. 
Our study had several limitations. This included the low number of patients $(n=15)$ and participating institutions $(n=3)$, with one facility responsible for a large proportion of patients $(10$ patients in one facility in 15 patients) and treatment courses (24 courses in one facility in 30 courses) available for analysis. As a result, TPE-specific parameters may be biased as to the number of procedures performed per TPE course or fluids and electrolyte replacement for each procedure. No significant difference in symptoms, procedures, and response to TPE was observed between patients with $(n=10)$ and without $(n=5)$ relapses except an average age of onset; 49.2 years old in patients with relapses vs. 27.8 years old in patients without relapses $(\mathrm{p}=0.0098)$. However, this result may be also due to limited number of patients. The difference between responders and non-responders to TPE was also not justified due to limited number of patients at this point. Therefore, our observations may change in the future, as data from more patients and facilities become available.

Based on these results, we are considering modification of our methods to collect more clinically useful and statistically meaningful data. In this regard, we are considering new methodology in standardizing and validating outcome measurements, which would allow confirmation of patient classification by response to TPE. We are also planning to investigate unique clinical features of MuSK MG patients, to identify potential risk factors in patient with multiple relapses, to identify impact of TPE on medication doses, and to identify the impact of TPE in long term management such as maintenance TPE.

In summary, this is the first multi-center registry study to evaluate demographic, TPE procedural and clinical data in MuSK MG patients who received TPE treatments. The overall subjective improvement was up to $94 \%$ after 4 TPE procedures; therefore, 5 TPE may be adequate as an initial course of TPE treatment for symptomatic exacerbation. However, 30.8\% 
of the patients were poor- or non-responders to TPE and $15.4 \%$ of the patients experienced diminished response over the time. We are considering modification of our methods to collect more practically useful and statistically meaningful data in the future based on our current data. Additional data from additional facilities will provide more accurate information in the context of the performance of TPE for this rare form of MG in the future.

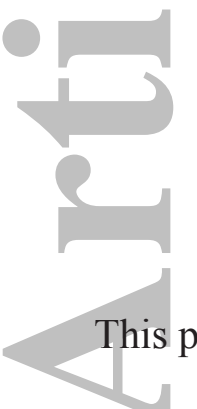

\section{Acknowledgement}

National Center for Advancing Translational Sciences. Its contents are solely the responsibility of the authors and do not necessarily represent the official views of the National Center for Advancing Translational Sciences or the National Institutes of Health. Additionally, we thank Lili Zhao, Ph.D., Assistant professor of Biostatistics at University of Michigan for her

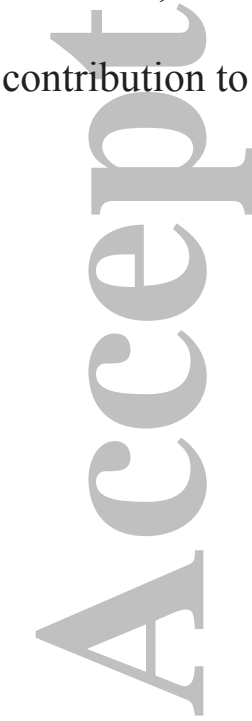




\section{References}

1. Hoch W, McConville J, Helms S, Newsom-Davis J, Melms A, Vincent A. Auto-antibodies to the receptor tyrosine kinase MuSK in patients with myasthenia gravis without acetylcholine receptor antibodies. Nat Med 2001;7:365-8.

2. Romi F, Gilhus NE, Aarli JA. Myasthenia gravis: clinical, immunological, and therapeutic advances. Acta Neurol Scand 2005;111:134-41.

3. Higuchi O, Hamuro J, Motomura M, Yamanashi Y. Autoantibodies to low-density lipoprotein receptor-related protein 4 in myasthenia gravis. Ann Neurol 2011;69:418-22.

4. Cossins J, Belaya K, Zoltowska K, Koneczny I, Maxwell S, Jacobson L, Leite MI, Waters P, Vincent A, Beeson D. The search for new antigenic targets in myasthenia gravis. Ann N Y Acad Sci 2012;1275:123-8.

5. Yamada C, Teener JW, Davenport RD, Cooling L. Maintenance plasma exchange treatment for muscle specific kinase antibody positive myasthenia gravis patients. J Clin Apher 2014;doi: 10.1002/jca.21377

6. Harris PA, Taylor R, Thielke R, Payne J Gonzalez N, Conde JG. Research electronic data capture (REDCap)--a metadata-driven methodology and workflow process for providing translational research informatics support. J Biomed Inform 2009;42:377-381

7. Reddel SW, Morsch M, Phillps WD. Clinical and scientific aspects of muscle-specific tyrosine kinase-related myasthenia gravis. Curr Opin Neurol 2014;27(5):558-65

8. Guptill JT, Sanders DB. Update on muscle-specific tyrosine kinase antibody positive myasthenia gravis. Curr Opin Neurol 2010;23(5):530-5.

9. Pasnoor M, Wolfe GI, Nations S, Trivedi J, Barohn RJ, Herbelin L, McVey A, Dimachkie M, Kissel J, Walsh R, Amato A, Mozaffar T, Hungs M, Chui L, Goldstein J, Novella S, Burns T, 
Phillips L, Claussen G, Young A, Bertorini T, Oh S. Clinical findings in MuSK-antibody positive myasthenia gravis: a U.S. experience. Muscle Nerve 2010;41:370-4.

0. Muppidi S, Wolfe GI. Muscle-specific receptor tyrosine kinase antibody-positive and seronegative myasthenia gravis. Front Neurol Neurosci 2009;26:109-19.

1. El-Salem K, Yassin A, Al-Hayk K, Yahya S, Al-Shorafat D, Dahbour SS. Treatment of MuSK-Associated Myasthenia Gravis. Curr Treat Options Neurol 2014;16:283.

2. Fraser ID, et al. What are the established clinical indications for therapeutic plasma exchange and how important is the choice of replacement fluid for efficacy of therapeutic plasma exchange in these situations? Vox Sang 1982;43(5):270-95

3. Madore F. Plasmapheresis technical aspects and indications. Crit Care Clin 2002;18:375-392

4. Couriel D, Weinstein R. Complications of therapeutic plasma exchange; a recent assessment. J Clin Apher 1994;9(1):1-5

5. Mokrzycki MH, Kaplan AA. Therapeutic plasma exchange: complications and management. Am K Kidney Dis 1994;23(6):817-27

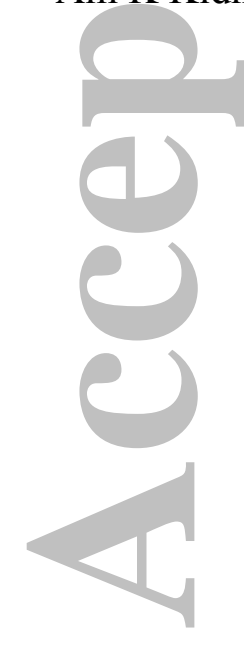


Table 1: Reported features of MuSK MG patients [1, 5, 8, 9, 10, 11]

1. Demographics

a. Predominantly female

b. Onset of symptoms in $4^{\text {th }}$ decade of life

2. Clinical course

a. More severe or refractory clinical symptoms

b. More rapidly progressive course with increased risk of myastenic crisis

3. Diagnostics

a. Lower diagnostic yield in repetitive nerve stimulation (RNS)

b. Myopathy-like electromyography (EMG)

c. Frequent abnormal single-fiber electromyography (SFEMG)

d. Normal thymus gland pathology

4. Treatment response

a. Poor response, more nicotinic and muscarinic side effects to anti-cholinesterase

b. Poor response to IVIG

c. Good response to immunosuppressive therapy

d. Good response to therapeutic plasma exchange (TPE)

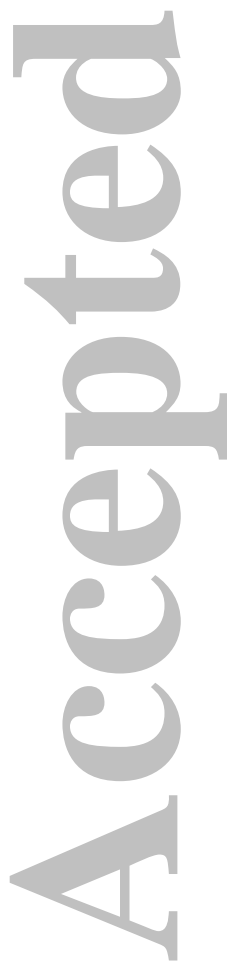




\section{Table 2: TPE procedural data on MuSK MG patients}

\section{Table 2A: based on courses}

\begin{tabular}{|c|c|c|c|c|}
\hline Parameter & $\begin{array}{c}\text { Reported } \\
\text { course number }\end{array}$ & Results & Courses & $\%$ \\
\hline Plasma volume exchanged & 30 & 1-1.25 total plasma volume & 30 & 100 \\
\hline Anticoagulant & 30 & ACD-A & 30 & 100 \\
\hline Rinse back & 30 & full & 30 & 100 \\
\hline Fluid balance & 30 & $100 \%$ & 30 & 100 \\
\hline \multirow[t]{8}{*}{ Total number of TPE in a course } & \multirow[t]{8}{*}{30} & 2 & 2 & 6.7 \\
\hline & & 3 & 5 & 16.7 \\
\hline & & 4 & 3 & 10.0 \\
\hline & & 5 & 14 & 46.7 \\
\hline & & 6 & 2 & 6.7 \\
\hline & & 7 & 2 & 6.7 \\
\hline & & 8 & 1 & 3.3 \\
\hline & & 10 & 1 & 3.3 \\
\hline \multirow[t]{4}{*}{ Frequency of TPE } & \multirow[t]{4}{*}{29} & every other day & 16 & 55.2 \\
\hline & & twice /week & 8 & 27.6 \\
\hline & & daily & 3 & 10.3 \\
\hline & & weekly & 2 & 6.9 \\
\hline \multirow[t]{4}{*}{ Access } & \multirow[t]{4}{*}{28} & peripheral veins & 14 & 50.0 \\
\hline & & central line (IJ) & 12 & 42.9 \\
\hline & & central line (SC) & 1 & 3.6 \\
\hline & & fistula & 1 & 3.6 \\
\hline
\end{tabular}

TPE: Therapeutic plasma exchange, MuSK: muscle specific kinase, MG: myasthenia gravis, ACD-A: anticoagulant citrate dextrose solution A, IJ: internal jugular vein, SC: subclavian vein, 
Table 2B: Based on procedures

\begin{tabular}{|c|c|c|c|c|}
\hline Parameter & $\begin{array}{c}\text { Reported } \\
\text { Procedure number }\end{array}$ & Results & Procedures & $\%$ \\
\hline \multirow{2}{*}{ Replacement fluid } & \multirow[t]{2}{*}{145} & $5 \%$ albumin only & 133 & 91.7 \\
\hline & & $5 \%$ albumin and normal saline & 12 & 8.3 \\
\hline \multirow[t]{3}{*}{ Calcium supplementation } & \multirow[t]{3}{*}{136} & added into albumin & 114 & 83.8 \\
\hline & & oral & 1 & 0.7 \\
\hline & & none & 21 & 15.4 \\
\hline Other supplementation & 145 & potassium, magnesium & 0 & 0 \\
\hline RBC priming & 145 & & 0 & 0 \\
\hline \multirow{5}{*}{ Adverse events } & \multirow[t]{5}{*}{145} & 5 procedures & & 3.4 \\
\hline & & citrate toxicities & 2 & 1.4 \\
\hline & & feeling of coldness & 1 & 0.7 \\
\hline & & palpitation and SOB & 1 & 0.7 \\
\hline & & hematoma at needle site & 1 & 0.7 \\
\hline
\end{tabular}

SOB: Shortness of breath

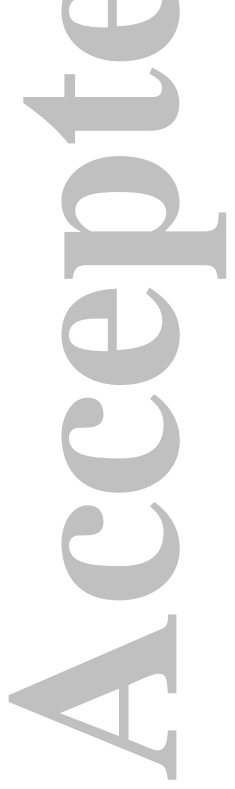

John Wiley \& Sons

This article is protected by copyright. All rights reserved. 
Table 3: Percentages of objective symptoms and full recovery of symptoms after a course of TPE

\begin{tabular}{lcrr}
\hline & Onset (n=15) & Before TPE (n=30) & Full recovery of symptom \\
\hline Diplopia & $93.3 \%(14 / 15)$ & $40.0 \%(12 / 30)$ & $83.3 \%(10 / 12)$ \\
\hline Ptosis & $40.0 \%(6 / 15)$ & $40.0 \%(12 / 30)$ & $75.0 \%(9 / 12)$ \\
\hline Dysarthria & $60.0 \%(9 / 15)$ & $66.7 \%(20 / 30)$ & $80.0 \%(16 / 20)$ \\
\hline Dysphagia & $73.3 \%(11 / 15)$ & $56.7 \%(17 / 30)$ & $94.1 \%(16 / 17)$ \\
\hline Weakness & $46.7 \%(7 / 15)$ & $46.7 \%(14 / 30)$ & $92.9 \%(13 / 14)$ \\
\hline SOB & $40.0 \%(6 / 15)$ & $40.0 \%(12 / 30)$ & $100.0 \%(2 / 2)$ \\
\hline Intubation & $13.3 \%(2 / 15)$ & $6.7 \%(2 / 30)$ & \\
\hline
\end{tabular}

Note: At onset, the percentage is based on total number of patients $(n=15)$. Before TPE, the percentage is based on total number of courses $(n=30)$. For full recovery of symptoms, the percentage is based on the number of course that patient had symptoms before TPE (numerator of before TPE in each symptom).

TPE: Therapeutic plasma exchange, SOB: Shortness of breath

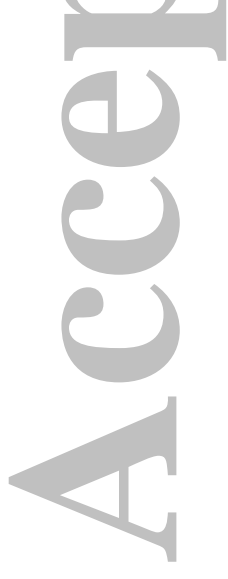


Table 4: Subjective improvement after a course of TPE

\begin{tabular}{|c|c|c|c|}
\hline Patient & Course & $\begin{array}{c}\text { Number of TPE } \\
\text { in a course }\end{array}$ & $\begin{array}{c}\text { Final subjective } \\
\text { improvement }\end{array}$ \\
\hline $1 C$ & 1 & 5 & Mild \\
\hline & 2 & 5 & No \\
\hline 2 & 3 & 3 & Moderate \\
\hline 3 & 4 & 4 & Mild \\
\hline 4 & 5 & 5 & Moderate \\
\hline 5 & 6 & 5 & \\
\hline & 7 & 4 & \\
\hline 6 & 8 & 5 & Moderate \\
\hline 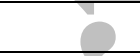 & 9 & 3 & Marked \\
\hline 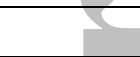 & 10 & 4 & Full \\
\hline 7 & 11 & 5 & Marked \\
\hline & 12 & 3 & Moderate \\
\hline$<<$ & 13 & 7 & \\
\hline & 14 & 6 & Mild \\
\hline & 15 & 5 & \\
\hline 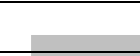 & 16 & 2 & Mild \\
\hline 8 & 17 & 5 & Full \\
\hline & 18 & 3 & Full \\
\hline 9 & 19 & 5 & Full \\
\hline 10 & 20 & 5 & \\
\hline 11 & 21 & 5 & Marked \\
\hline 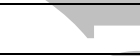 & 22 & 5 & Marked \\
\hline 7 & 23 & 5 & Marked \\
\hline 12 & 24 & 5 & Marked \\
\hline 13 & 25 & 10 & Marked \\
\hline & 26 & 8 & Moderate \\
\hline & 27 & 7 & Mild \\
\hline C & 28 & 6 & No \\
\hline 14 & 29 & 3 & Mild \\
\hline 15 & 30 & 2 & No \\
\hline
\end{tabular}

TPE: Therapeutic plasma exchange 
Table 5: Percentage of subjective improvement after each TPE

\begin{tabular}{|c|c|c|c|c|c|}
\hline & After $1^{\text {st }}$ TPE & After $2^{\text {nd }}$ TPE & After $3^{\text {rd }}$ TPE & After $4^{\text {th }}$ TPE & $\begin{array}{c}\text { After }>5 \text { TPE } \\
\text { (at the end of course) }\end{array}$ \\
\hline Number of reported courses & 25 & 21 & 17 & 15 & 5 \\
\hline No improvement & $36.0 \%(9 / 25)$ & $19.0 \%(4 / 21)$ & $5.9 \%(1 / 17)$ & $6.7 \%(1 / 15)$ & $20.0 \%(1 / 5)$ \\
\hline Mild improvement & $40.0 \%(10 / 25)$ & $42.9 \%(9 / 21)$ & $35.3 \%(6 / 17)$ & $20.0 \%(3 / 15)$ & $40.0 \%(2 / 5)$ \\
\hline Moderate improvement & $16.0 \%(4 / 25)$ & $28.6 \%(6 / 21)$ & $29.4 \%(5 / 17)$ & $20.0 \%(3 / 15)$ & $20.0 \%(1 / 5)$ \\
\hline Marked improvement & $8 \%(2 / 25)$ & $0 \%(0 / 21)$ & $29.4 \% 5 / 17)$ & $40.0 \%(6 / 15)$ & $20.0 \%(1 / 5)$ \\
\hline Full improvement & $0 \%(0 / 25)$ & $9.5 \%(2 / 21)$ & $0 \%(0 / 17)$ & $13.3 \%(2 / 15)$ & $0 \%(0 / 5)$ \\
\hline Any improvement & $64.0 \%(16 / 25)$ & $80.1 \%(17 / 21)$ & $94.1 \%(16 / 17)$ & $93.3 \%(14 / 15)$ & $80.0 \%(4 / 5)$ \\
\hline
\end{tabular}

TPE: Therapeutic plasma exchange

Note: The occurrence percentage of improvement is based on the number of reported courses in each evaluation time.

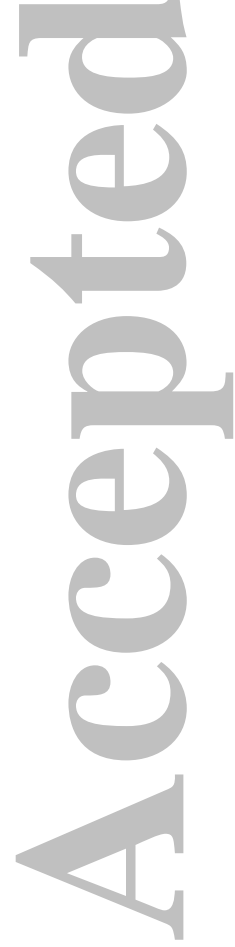


Table 6: Pre-procedure medications

\begin{tabular}{lrr}
\hline Medication & 30 courses & \% \\
\hline none & 6 & 20.0 \\
\hline prednisone & 17 & 56.7 \\
\hline hydrocortisone & 1 & 3.3 \\
\hline methylprednisone & 0 & 0 \\
\hline pyridostigmine & 13 & 43.3 \\
\hline IVIG & 3 & 10.0 \\
\hline rituximab & 1 & 3.3 \\
\hline azathioprine & 3 & 10.0 \\
\hline mycophenolate mofetil & 1 & 3.3 \\
\hline cyclosporine & 0 & 0 \\
\hline
\end{tabular}

John Wiley \& Sons

This article is protected by copyright. All rights reserved. 
Table 7: Change of medication after a course of TPE

7A: Prednisone

\begin{tabular}{|l|r|r|r|r|}
\hline \multirow{2}{*}{} & \multicolumn{2}{|c|}{ Right after (14 courses) } & \multicolumn{2}{|c|}{ 3 months after (13 courses) } \\
\cline { 2 - 5 } & Courses & $\mathbf{\%}$ & Courses & \% \\
\hline Decreased & 1 & 7.1 & 4 & 30.8 \\
\hline Increased & 2 & 14.3 & 0 & 0 \\
\hline No change & 11 & 78.6 & 9 & 69.2 \\
\hline
\end{tabular}

7B: Pyridostigmine

\begin{tabular}{|l|r|r|r|r|}
\hline & \multicolumn{2}{|c|}{ Right after (6 courses) } & \multicolumn{2}{|c|}{ 3 months after (5 courses) } \\
\cline { 2 - 5 } & Courses & \% & Courses & \% \\
\hline Decreased & 4 & 66.7 & 4 & 80.0 \\
\hline Increased & 0 & 0 & 0 & 0 \\
\hline No change & 2 & 33.3 & 1 & 20.0 \\
\hline
\end{tabular}

TPE: Therapeutic plasma exchange 